\title{
NEUROPHYSIOLOGICAL FEATURES OF THE NOCICEPTIVE TRIGEMINAL PATHWAY IN ABDOMINALLY OBESE MIGRAINEURS
}

D0I: 10.36740/WLek202004109

\author{
Olena S. Nikiforova, Mykhaylo Yu. Delva \\ UKRAINIAN MEDICAL STOMATOLOGICAL ACADEMY, POLTAVA, UKRAINE
}

\begin{abstract}
The aim of this study were to assess clinical (cutaneous allodynia) and neurophysiological (R2 nBR component) markers of the nociceptive trigeminal-cervical pathway sensitization in the abdominally obese patients with episodic (EM) and chronic migraine (CM).

Materials and methods: It had been recorded nBR within interictal period in 79 migraineurs with EM and CM who had abdominal obesity (A0) and normal body weight (NBW).

Results: Patients with EM and A0 had significantly lower pain thresholds than patients with EM and NBW - 0,55 (0,34-0,63) $\mu \mathrm{V}$ vs $0,70(0,59-1,03) \mu \mathrm{V}$. The mean latency of R2 nBR component was significantly decreased in patients who had EM and A0 (36,59 (31,95-42,41) ms) compared to patients who had EM and NBW(46,75 (42,93-52,34) ms). It had been revealed significant increasing of the mean amplitude of the component R2 nBR in patients who had EM and A0 (158 (115-197) $\mu \mathrm{V}$ ) compared to patients who had EM and NBW (124 (76-144) $\mu V)$. In patients who had $C M$ and $A 0$, it had been found direct positive correlation between the amplitude of $R 2 n B R$ and severity of cutaneous allodynia $-\tau=0,4(p=0,03)$, as well as found negative correlation between the latency of $R 2 n B R$ and severity of cutaneous allodynia $-\tau=-0,44(p=0,02)$ within the interictal period of migraine.

Conclusions: Neurophysiological findings may indirectly indicate faster migraine chronification in patients with EM and A0.
\end{abstract}

KEY WORDS: Chronic migraine, Episodic migraine, Abdominal obesity, Nociceptive blink reflex, Trigeminal pathway

\section{INTRODUCTION}

Migraine is one of the most common types of primary headache. General population studies have confirmed its high prevalence as well as its high socio-economic and personal burden [1]. According to recent data, migraine is the third most common disorder and the seventh-largest specific cause of disability worldwide [1].

Migraine - is a chronic relapsing disease with episodic manifestations and ability to progress in people who have high genetic predisposition or high susceptibility to chronic exposure of risk factors [2]. As important risk factor for the progression of episodic migraine (EM) and subsequent conversion of EM into chronic migraine (CM) - in case of paroxysms' frequency increasing up to 15 or more per month, is considered abdominal obesity ( $\mathrm{AO}$ ) [3migraine and tension-type headache,4] transformed migraine (TM. On the other hand, $\mathrm{AO}$ is of particular importance due to the fact that it can be effectively modified.

Despite the fact that headache is the most striking clinical sign, migraine is primarily a sensory processing disorder. It is considered that in the chronicity of migraine headache is involved activation of the trigeminovascular system and sensitization of the trigeminal nucleus in the brainstem, as well as disturbances of the central inhibitory antinociceptive control mechanisms.

Sensitization is a complex extended in time process: decreasing the intensity of stimulus which is necessary for the formation of a response and increasing the amplitude of the reaction to a given stimulus [5]. Cutaneous allodynia is a sensation of pain or discomfort due to irritation of ordinary thermal (heat or cold) and/or mechanical (static or dynamic) stimuli on normal skin [6]. Nowadays, cutaneous allodynia is considered as a clinical marker of central sensitization as well as an independent predictor of migraine chronification, that can be easily identified $[7,8] 000$ headache sufferers previously identified from the population. The questionnaire included the validated Allodynia Symptom Checklist (ASC.

Neurophysiological tests, including studies of the nociceptive blink reflex (nBR), had become an important tool for investigation of cerebral irritability, central and peripheral mechanisms of perception and chronicity of pain [9]. The study of $\mathrm{nBR}$ is an objective non-invasive quantitative neurophysiological method used to assess the functions of the trigeminal system because the afferent part of the reflex arc is represented with nociceptive trigeminal fibers [10]. This reflex is obtained by special stimulating concentric electrode by means of supraorbital nerve stimulation on one side of the face, with subsequent ipsilateral and contralateral responses [11].

\section{THE AIM}

The objectives of this study were to assess the clinical (cutaneous allodynia) and neurophysiological (characteristics of R2 nBR component) markers of the nociceptive trigeminal-cervical pathway sensitization in the abdominally obese patients with EM and CM. 


\section{MATERIALS AND METHODS}

After signing the informed consent, the study included patients of both genders aged from 18 to 65 years with migraine (according to ICHD-3, 2019) who had normal body weight (NBW) - the value of the body mass index less than $25 \mathrm{~kg} / \mathrm{m} 2$ and $\mathrm{AO}$. AO was diagnosed in case of waist circumferences more than $102 \mathrm{~cm}$ in men and more than $88 \mathrm{~cm}$ in women [12].

Depending on the frequency of migraine attacks, it had been formed patient group who had EM (less than 15 a month) and patient group who had CM (15 or more a month). We recruited 20 patients with EM and NBW, 19 patients with EM and $\mathrm{AO}, 19$ patients with $\mathrm{CM}$ and normal body weight, and 21 patients with $\mathrm{CM}$ and $\mathrm{AO}$.

Data was collected directly in contact with patients using the structured questionnaire, which included data about disease duration (years), frequency of migraine attacks per month), and average duration of migraine attacks (hours). Throughout the socio-demographic characteristics of patients, the age and gender of the patients were analyzed. Quantitative and qualitative characteristics of cutaneous allodynia (mechanical, dynamic, thermal) were studied using the ASC-12 questionnaire [13] and to estimate the prevalence and severity of CA in the migraine population. METHODS: Migraineurs $(\mathrm{n}=11,388$.

In order to study the possible dysfunctions of neurons in the brainstem, the specific $\mathrm{nBR}$ component $\mathrm{R} 2$ was studied. $n B R$ was illicited by selective stimulation of the A- $\delta$ fibers of the supraorbital nerve (without depolarization of the nociceptive A- $\beta$ fibers in the deeper layers of the skin) by special electrode with a high current density (inomed Medizintechnik GmbH, Emmendingen, Germany, http:// www.inomed.com) [11].

Exclusion criteria for all patient groups were: the age under 18 years or above 65 years, the presence of peripheral facial or trigeminal neuropathy, dermatological diseases, clinical depression and anxiety (the subscale value of the Hospital Anxiety and Depression Scale is 11 or more points [14]), severe drug dependence (the value of the LDQ questionnaire is 23 or more points [15] by the Leeds Dependence Questionnaire (LDQ), botulinum toxin treatment for the past three months.

\section{EXPERIMENTAL PROCEDURE}

The $n B R$ evaluation was performed at the same time of the day (between 13.00-16.00) using «Neurosoft» MEP-4 electroneuromyography (LTD «Neurosoft», Russia, Ivanovo) within the interictal period: at least two days after the last attack, but for patients with CM a slight chronic headache was allowed. The patient was supine with his eyes closed during recording. Threshold values of sensory and pain perception were defined as the minimum stimulation intensity that caused tactile and pain sensation, respectively. First, we determined sensory and pain thresholds in the supraorbital region on both sides using a single ascending impulse with a step of $0.1 \mathrm{~mA}$, a duration of $0.2 \mathrm{~ms}$, and interval between stimulations of 15-20 s (randomized interval).
For the patient's comfort and for shortening of procedure duration, we provided one-sided stimulation. The concentric electrode was placed in the area of the supraorbital region, approximately $10 \mathrm{~mm}$ above the foramen supraorbialae, to stimulate the supraorbialae nerve on the right. An electric stimulus intensity of $1.5 \times$ individual pain threshold value was used to induce nBR. We did not use stimulation intensity more than $2 \mathrm{~mA}$ in order to minimize the risk of A- $\beta$ fibers activation [11]. Stimulation was repeated after the next $20 \mathrm{~s}$ (randomized inter-pulse interval) to obtain at least 11 consecutive responses. The first recording was excluded from the analysis as possible startle response. Surface discharge electrodes for $\mathrm{nBR}$ recording were placed in the infraorbital region (active) and in the nasal back area (reference). For recording were used the following characteristics: sampling rate $-2.5 \mathrm{kHz}$, sweep length $-7.5 \mathrm{~ms}$, analysis time -200 $\mathrm{ms}$ and sensitivity $-75 \mu \mathrm{V}$.

The following parameters were measured of the ipsilateral and contralateral R2 $\mathrm{nBR}$ component: latency (ms), mean amplitude $(\mu \mathrm{V})$, area under the curve (using ImageJ in pixels), habitat index (as the percentage change of the R2 area of the first block compared to the tenth block of recordings).

In the analysis of quantitative values, the normality of their distribution was checked using the Kolmogorov-Smirnov test. Quantitative values had a nonparametric distribution and were presented as median $(\mathrm{Me})$ and interquartile (25\%-75\%) range (Q1-Q3). Significant differences between quantitative values was performed using the Mann-Whitney paired U-test (between two independent groups). Correlations were estimated using the $\tau$ Kendall rank correlation coefficient. A $p$-value $<0,05$ was considered as statistically significant.

\section{RESULTS}

Among patients with $\mathrm{CM}$ and $\mathrm{AO}$, compared with patients with CM and normal body weight, it had been found a significantly shorter duration of the disease - $10(8-15)$ years vs. $8(6-10)$ years $(\mathrm{p}=0.03)$, and significantly lower age -38 $(31-46)$ years vs. $31(28-36)$ years $(p=0.03)$, respectively. These peculiarities may indirectly indicate faster migraine chronification in abdominally obese patients.

Table 1 demonstrates that patients with EM and AO had significantly lower pain thresholds than patients with EM and NBW $(\mathrm{p}=0.03)$ and the later had pain thresholds almost equal to pain thresholds in patients with $\mathrm{CM}$.

Table 1. The values of pain thresholds depending on the type of migraine and the presence of $\mathrm{AO}, \mathrm{Me}(\mathrm{Q1}-\mathrm{Q3})$

\begin{tabular}{cc}
\hline Groups of patients & Pain thresholds, $(\boldsymbol{\mu} \mathbf{V})$ \\
\hline EM, NBW & $0,70(0,59-1,03)$ \\
\hline CM, NBW & $0,61(0,48-0,83)$ \\
\hline EM, AO & $0,55(0,34-0,63)^{*}$ \\
\hline CM, AO & $0,72(0,49-0,91)$ \\
\hline
\end{tabular}

* - significant differences, according to the Mann-Whitney U test, compared with patients who had EM and NBW. 
Table 2. Parameters of R2 nBR component, Me (Q1-Q3)

\begin{tabular}{cccc}
\hline \multirow{2}{*}{ Group of patients } & \multicolumn{3}{c}{ Parameters of $\mathbf{R} \mathbf{2} \mathbf{n B R}$} \\
\cline { 2 - 4 } & latency, $\mathbf{m s}$ & amplitude, $\boldsymbol{\mu} \mathbf{V}$ & duration, $\mathbf{m s}$ \\
\hline EM, NBW & $46,75(42,93-52,34)$ & $124(76-144)$ & $46,7(45,8-52,3)$ \\
\hline CM, NBW & $49,42(39,27-54,52)$ & $85(71-110)^{*}$ & $48,3(41,9-49,6)$ \\
\hline EM, AO & $36,59(31,95-42,41)^{*}$ & $158(115-197)^{*}$ & $49,2(40,7-50,8)$ \\
\hline CM, AO & $52,84(37,21-56,54)$ & $93(62-118)^{* * *}$ & $42,6(39,0-50,7)$ \\
\hline
\end{tabular}

* - significant differences, according to the Mann-Whitney $U$ test, compared with patients who had EM and NBW;

** - significant differences, according to the Mann-Whitney U test, compared with patients who had EM and A0.

Table 3. The value of the habituation index, Me (Q1-Q3)

\begin{tabular}{cc}
\hline Group of patients & Habituation index \\
\hline EM, NBW & $0,87(0,82-0,94)$ \\
\hline CM, NBW & $0,41(0,26-0,48)$ \\
\hline EM, AO & $0,58(0,32-0,84)^{*}$ \\
\hline CM, AO & $0,49(0,29-0,74)$ \\
\hline
\end{tabular}

* - significant differences, according to the Mann-Whitney U test, compared with patients who had EM and NBW.

As can be seen from Table 2, the mean latency of $\mathrm{R} 2 \mathrm{nBR}$ component was significantly decreased in patients who had EM and AO compared with patients who had EM and NBW. Whereas no significant difference was observed in the groups with $\mathrm{CM}$, regardless of body weight.

The mean amplitude of the $\mathrm{R} 2 \mathrm{nBR}$ component was significantly higher in the EM patients' groups compared to the CM patient's groups, regardless of anthropometric characteristics. Additionally, it had been revealed a significant increasing of the mean amplitude of the component $\mathrm{R} 2 \mathrm{nBR}$ in patients who had EM and AO compared with patients who had EM and NBW.

In all patient's groups, there were no significant differences in the mean duration of the R $2 \mathrm{nBR}$ component.

As can be seen from table 3, habituation deficit, as a specific electrophysiological symptom of EM within the interictal period, was not observed in patients with $\mathrm{AO}$. In the contrary, normal rates of habituation were recorded, which is characteristic of CM and ictal periods of EM.

In patients who had $\mathrm{CM}$ and $\mathrm{AO}$, it had been found direct positive correlation between the amplitude of $\mathrm{R} 2 \mathrm{nBR}$ and severity of cutaneous allodynia $-\tau=0,4(p=0,03)$, as well as found negative correlation between the latency of R $2 \mathrm{nBR}$ and severity of cutaneous allodynia $-\tau=-0,44(p=0,02)$ within the interictal period of migraine.

\section{DISCUSSION}

This is the first study that provides electrophysiological evidence about possible alteration of nociceptive trigeminal pathway in the brainstem in abdominally obese patients with migraine.

The neural circuit mediated by the $\mathrm{R} 2$ component $\mathrm{nBR}$ includes supraorbital nerve, trigeminal caudal nucleus, excitatory interneurons of reticular formation, facial motor nuclei and facial nerve that innervate the circular muscles of the eye [16]. Central sensitization of trigeminal spinal nuclei causes depolarization of cutaneous trigeminal axons and, as a consequence, changes in R2 component nBR. [17]. Response of $\mathrm{R} 2$ component $\mathrm{nBR}$ is modulated by segmental and supra-segmental mechanisms [18]. The lowered pain thresholds within interictal periods in individuals who had $\mathrm{EM}$ and $\mathrm{AO}$, may indicate some modulation of descending pain system due to antinociceptive deficiency.

We observed significant differences of $\mathrm{R} 2$ component parameters in patients with EM and $\mathrm{AO}$. Latency decreasing and amplitude increasing of $\mathrm{R} 2 \mathrm{nBR}$ component can be considered as facilitation in trigeminal nociceptive system. Facilitation of trigeminal nociceptive system likely is associated with migraine-specific brainstem hypersensitivity [19]. According to our findings, facilitation of trigeminal nociceptive system is most pronounced in patients with $\mathrm{AO}$, and it can be ground for more frequent migraine paroxysms occurrence. Neurophysiological reactions during episodic headaches at first are adaptive and physiological, but in process of time they become maladaptive and pathological, eventually creating a vicious circle for chronic headache development [20]. Headache evolution process is associated with neural plasticity of brain excitability, with biochemical, functional and even structural changes [21]. Comorbid conditions, in particular $\mathrm{AO}$, contribute to this process [22].

Habituation is a phenomenon of reduced response after repeated stimulation [23]. Habituation is a common physiological feature of responses to any type of sensory stimulation. In terms of algology, habituation helps to save a healthy balance between nociceptive and antinociceptive systems. According to previous studies, phenomenon of habituation deficit was observed in the interictal phases of EM $[24,25]$ and it may represent a basic problem of sensory perception and processing in patients with migraine [26]. According to our findings, abdominally obese patients hadn't habituation deficit within interictal periods of EM, on the contrary, they had normal indicators of habituation, which are typical for CM and ictal periods of EM [27], so it can be described as follows - «the attack that cannot stop». Also it had been suggested, that habituation changes may be associated with transforming of EM into CM [28], and $\mathrm{AO}$ can be actively involved it these processes.

According to our study, metabolic disorders in particular, local and systemic inflammation, that are related to $\mathrm{AO}$ [29], 
may influence on the brainstem sensory and supra-segmental processing and in this manner contribute to central sensitization and accelerate migraine chronification.

\section{CONCLUSIONS}

1. Patients with AO are significantly younger and have significantly shorter migraine duration, compared to normal body weight patients that may indirectly indicate faster migraine chronification.

2. Episodic migraineurs with $\mathrm{AO}$ have some neurophysiological abnormalities compared to normal body weight migraineurs (significantly lower pain thresholds, amplitude increasing and latency decreasing of the $\mathrm{R} 2$ component $\mathrm{nBR}$ within interictal periods, normal habituation indicators).

3. Patients with $\mathrm{CM}$ and $\mathrm{AO}$ have reliable correlations between R2 nBR characteristics and cutaneous allodynia severity.

Perspectives for future research. These results may be useful for better understanding the role of trigeminal complex in the pathophysiology of headache, for monitoring patients during migraine episodes, or evaluating response to the treatment. The greater contribution of electrophysiological testing could be helpful for identification of migraineurs groups and could serve as a strategy for personalized migraine treatment.

\section{REFERENCES}

1. GBD 2015 Disease and Injury Incidence and Prevalence Collaborators Global, regional, and national incidence, prevalence, and years lived with disability for 310 diseases and injuries 1990-2015: a systematic analysis for the Global Burden of Disease Study 2015. Lancet. 2016;388:1545-1602.

2. Lipton R.B., Bigal M.E. Migraine: epidemiology, impact, and risk factors for progression. Headache. 2005;45(1):3-13.

3. Robberstad L., Dyb G., Hagen K. et al. An unfavorable lifestyle and recurrent headaches among adolescents: the HUNT study. Neurology. 2010;75(8):712-717.

4. Bigal M.E., Lipton R.B. Obesity is a risk factor for transformed migraine but not chronic tension-type headache. Neurology. 2006;67(2):252-257.

5. Perrotta A., Serrao M., Sandrini G., et al. Sensitisation of spinal cord pain processing in medication overuse headache involves supraspinal pain control. Cephalalgia. 2010;30(3):272-284.

6. Tietjen G.E., Brandes J.L., Peterlin B.L. et al. Allodynia in migraine: association with comorbid pain conditions. Headache. 2009 0ct;49(9):1333-1344.

7. Bigal M.E., Ashina S., Burstein R. et al. Prevalence and characteristics of allodynia in headache sufferers: a population study. Neurology. 2008;70(17):1525-1533.

8. Aguggia M. Allodynia and migraine. Neurological sciences: official journal of the Italian Neurological Society and of the Italian Society of Clinical Neurophysiology. 2012;33(1):9-11.

9. Filatova E., Latysheva N., Kurenkov A. Evidence of persistent central sensitization in chronic headaches: A multi-method study. J Headache Pain. 2008;9(5):295-300.

10. Marin J.C., Gantenbein A.R., Paemeleire K. et al. Nociception-specific blink reflex: pharmacology in healthy volunteers. J Headache Pain. 2015;16(1). http://dx.doi.org/10.1186/s10194-015-0568-7
11. Kaube H., Katsarava Z., Kaufer T. et al. A new method to increase nociception specificity of the human blink reflex. Clin Neurophysiol. 2000;111(3):413-416.

12. World Health Organization (WHO). Waist Circumference and Waist-Hip Ratio: Report of a WHO Expert Consultation. Geneva: World Health Organization; 2008.

13. Lipton R.B., Bigal M.E., Ashina S. et al. Cutaneous allodynia in the migraine population. Ann Neurol. 2008;63(2):148-158.

14. Zigmond A.S., Snaith R.P. The hospital anxiety and depression scale. Acta Psychiatr Scand. 1983;67(6):361-370.

15. Ferrari A., Cicero A.F., Bertolini A. et al. Need for analgesics/drugs of abuse: a comparison between headache patients and addicts by the Leeds Dependence Questionnaire (LDQ). Cephalalgia. 2006;26(2):187-193.

16. Ellrich J. Brain Stem Reflexes: Probing Human Trigeminal Nociception. News Physiol Sci an Int J Physiol Prod jointly by Int Union Physiol Sci Am Physiol Soc. 2000;15:94-97.

17. Esteban A. A neurophysiological approach to brainstem reflexes. Blink reflex. Neurophysiol Clin. 1999;29(1):7-38.

18. Valls-Sole J. Assessment of excitability in brainstem circuits mediating the blink reflex and the startle reaction. Clin Neurophysiol. 2012;123(1):13-20.

19. Katsarava Z., Lehnerdt G., Duda B. et al. Sensitization of trigeminal nociception specific for migraine but not pain of sinusitis. Neurology. 2002;59(9):1450-1453.

20. Borsook D., Maleki N., Becerra L. et al. Understanding migraine through the lens of maladaptive stress responses: a model disease of allostatic load. Neuron. 2012;73(2):219-234.

21. Welch K.M. Contemporary concepts of migraine pathogenesis. Neurology. 2003;61(8):2-8.

22. Bigal M.E., Rapoport A.M. Obesity and chronic daily headache. Curr Pain Headache Rep. 2012;16(1):101-109.

23. Ferraro D., Vollono C., Miliucci R. et al. Habituation to Pain in"Medication Overuse Headache": A C02 Laser-Evoked Potential Study. Headache J Head Face Pain. 2012;52(5):792-807.

24. Katsarava Z., Giffin N., Diener H.C. et al. Abnormal habituation of "nociceptive" blink reflex in migraine--evidence for increased excitability of trigeminal nociception. Cephalalgia. 2003;23(8):814-819.

25. De Marinis M., Pujia A., Natale L. et al. Decreased habituation of the R2 component of the blink reflex in migraine patients. Clin Neurophysiol. 2003;114(5):889-893.

26. Di Clemente L., Coppola G., Magis D. et al. Interictal habituation deficit of the nociceptive blink reflex: an endophenotypic marker for presymptomatic migraine? Brain. 2007;130(3):765-770.

27. de Tommaso M., Murasecco D., Libro G. et al. Modulation of trigeminal reflex excitability in migraine: effects of attention and habituation on the blink reflex. Int J Psychophysiol. 2002;44(3):239-349.

28. Filatova E., Latysheva N., Kurenkov A. Evidence of persistent central sensitization in chronic headaches: a multi-method study. J Headache Pain. 2008;9(5):295-300.

29. Smith U. Abdominal obesity: a marker of ectopic fat accumulation. J Clin Invest. 2015;125(5):1790-1792.

The research described in this paper was performed according to scientific plan of neurological department with neurosurgery and medical genetics at Ukrainian medical stomatological academy "Clinical and pathogenetic optimization of diagnosis, prognosis, treatment and prevention of complicated central nervous system's disorders and neuro- 
logical impairments due to therapeutic pathologies" (state registration number 0116U004190).

\section{ORCID and contributionship:}

Olena S. Nikiforova - 0000-0003-1775-6050 A,D,E

Mykhaylo Yu. Delva - 0000-0001-5648-7506 $6^{B, C, F}$

\section{Conflicts of interest:}

Authors declare no conflict of interest.

\section{CORRESPONDING AUTHOR}

\section{Olena S. Nikiforova}

Ukrainian Medical Stomatological Academy

Shevchenko 23, 36001, Poltava, Ukraine

tel:+380669329779

e-mail: elenanikvor@gmail.com

Received: 28.10 .2019

Accepted: 24.02 .2020

A - Work concept and design, B - Data collection and analysis, C - Responsibility for statistical analysis,

D-Writing the article, $\mathbf{E}$ - Critical review, $\mathbf{F}$ - Final approval of the article 